\title{
Theoretical mass sensitivity of Love wave and layer guided acoustic plate mode sensors
}

\author{
G. McHale, ${ }^{\text {a) }}$ M. I. Newton, and F. Martin \\ Department of Chemistry and Physics, The Nottingham Trent University, Clifton Lane, \\ Nottingham, NG11 8NS, United Kingdom
}

(Received 30 January 2002; accepted for publication 20 March 2002)

\begin{abstract}
A model for the mass sensitivity of Love wave and layer guided shear horizontal acoustic plate mode (SH-APM) sensors is developed by considering the propagation of shear horizontally polarized acoustic waves in a three layer system. A dispersion equation is derived for this three layer system and this is shown to contain the dispersion equation for the two layer system of the substrate and the guiding layer plus a term involving the third layer, which is regarded as a perturbing mass layer. This equation is valid for an arbitrary thickness perturbing mass layer. The perturbation, $\Delta \nu$, of the wave speed for the two-layer system by a thin third layer of density, $\rho_{p}$ and thickness $\Delta h$ is shown to be equal to the mass per unit area multiplied by a function dependent only on the properties of the substrate and the guiding layer, and the operating frequency of the sensor. The independence of the function from the properties of the third layer means that the mass sensitivity of the bare, two-layer, sensor operated about any thickness of the guiding layer can be deduced from the slope of the numerically or experimentally determined dispersion curve. Formulas are also derived for a Love wave on an infinite thickness substrate describing the change in mass sensitivity due to a change in frequency. The consequences of the various formulas for mass sensing applications are illustrated using numerical calculations with parameters describing a (rigid) poly(methylmethacrylate) wave-guiding layer on a finite thickness quartz substrate. These calculations demonstrate that a layer-guided SH-APM can have a mass sensitivity comparable to, or higher, than that of Love waves propagating on the same substrate. The increase in mass sensitivity of the layer guided SH-APMs over previously studied SH-APM sensors is of significance, particularly for liquid sensing applications. The relevance of the dispersion curve to experiments using higher frequencies or frequency hopping and to experiments using thick guiding layers is discussed. (C) 2002 American Institute of Physics. [DOI: 10.1063/1.1477603]
\end{abstract}

\section{INTRODUCTION}

Acoustic wave sensors work on the simple principle that a surface is set into high frequency oscillation and interactions with the environment close to the surface cause either energy storage or energy loss. These effects are often observed experimentally as changes in resonant frequency, representing a shift in wave speed, and as a broadening of a resonance frequency, representing changes in attenuation. In Rayleigh surface acoustic wave (SAW) sensors, the substrate particles execute a retrograde elliptical motion in the plane described by the direction of propagation and the normal to the surface. ${ }^{1}$ Rayleigh-SAWs are highly sensitive to deposited surface mass but, due to the out-of-plane component of their displacement have significant attenuation if the surface supporting the wave (i.e., the sensing surface) is exposed to a liquid. This is unfortunate since many applications of current interest involve the deposition of mass from the liquid phase. There are two types of approach to extending the use of acoustic wave sensors to the liquid phase. The first is to use a flexural plate wave which, although it has an out-of-plane component of displacement, has a wave speed less than the

\footnotetext{
a) Author to whom correspondence should be addressed; electronic mail: glen.mchale@ntu.ac.uk
}

speed of sound in the liquid. ${ }^{2,3}$ Under these circumstances, the wave can no longer generate compressional waves in the liquid and so does not suffer a large attenuation. The second approach is to use an acoustic wave mode that has surface parallel displacements. Examples of such waves, in approximate order of mass sensitivity, include the thickness shear mode of the quartz crystal microbalance, ${ }^{4,5}$ a shear horizontal acoustic plate mode (SH-APM) ${ }^{6-8}$ a shear horizontal surface acoustic wave (SH-SAW), ${ }^{9}$ a layer-guided SH-SAW, ${ }^{10}$ a surface transverse wave, ${ }^{11}$ or a Love wave, ${ }^{12-14}$ which is a SH mode with a wave guiding layer. ${ }^{15}$ The difference in the sensitivity between these devices is enormous with the mass sensitivity of Love waves, and more recently the layer guided SH-SAWs, being several orders of magnitude greater than that of SH-APMs. For this reason much recent experimental work has preferred Love wave devices. However, a problem with such devices is that the interdigital transducers (IDTs) used to generate and detect the wave are located on the same face of the substrate as the contacting liquid; this is a problem not shared by the less sensitive SH-APM devices. Locating IDTs on the same side as the liquid causes difficulties because of the dielectric constant of the liquid and, depending on the guiding layer's dielectric constant, the need to have liquid seals within the propagation path. 
In this work, we develop a theoretical framework for understanding the mass sensitivity of layer guided $\mathrm{SH}-\mathrm{APM}^{16,17}$ and Love wave sensors. In Sec. II, the propagation of a shear horizontally polarized acoustic wave in a three-layer system is considered and a dispersion equation is derived. In Sec. III, the third layer is then regarded as a thin mass layer that is to be sensed and a perturbation approach is used to derive a mass sensitivity formula for the phase speed. The basic sensor characteristics of a layer guided acoustic wave device can be deduced from the dispersion equation describing the substrate and wave-guiding layer. Section IV develops, quantitatively, the interpretation of this dispersion equation and introduces the idea of determining the mass sensitivity from a numerically or experimentally determined dispersion curve. In Sec. V, the consequences of the relationship between the mass sensitivity and the dispersion curve are derived for a device operated at either a higher frequency or more than one frequency. The previous formulas are then considered numerically and their predictions for sensors and their consequences for current experiments are discussed in Sec. VI. The relevance of the theory to Love wave sensors working at multiple frequencies and to Love wave sensors with thick wave-guiding layers with lossy materials is discussed. It is also suggested that the layer guided SH-APM sensor will have a significantly enhanced mass sensitivity, possibly exceeding that of a Love wave using the same substrate and guiding layer. This suggestion alters the currently accepted notions of the most sensitive type of device and offers the advantage of a highly mass sensitive liquid phase sensor with the transducers located on the opposite face to the sensing surface.

\section{THEORETICAL FORMULATION}

The problem of the response of a two-layer system of a substrate and a waveguide to the deposition of rigid mass is essentially the problem of the propagation of acoustic waves in a three-layer system. For the finite substrate Love wave and layer guided SH-APM sensors, we consider a substrate of thickness, $w$, with a density $\rho_{s}$ and Lamé constants $\lambda_{s}$ and $\mu_{s}$ overlayed by a uniform mass layer of thickness, $d$, and with a density $\rho_{l}$ and Lamé constants $\lambda_{l}$ and $\mu_{l}$. In analogy to Love wave theory, the uniform mass overlayer is referred to as the guiding layer; this two-layer system is the bare sensor and possesses a dispersion curve. In a previous report ${ }^{17}$ we described how a dispersion equation can be derived for this two-layer system and how that dispersion equation contains generalisations of Love waves from infinite thickness substrates to finite thickness substrate and of acoustic plate modes from nonguided to layer guided modes. The present formulation uses the same approach, but introduces into the system a third layer of thickness, $h$, with a density $\rho_{p}$, and Lamé constants $\lambda_{p}$ and $\mu_{p}$. This third layer is referred to as a perturbing mass layer, although the dispersion equation derived in this section for the three-layer system is in fact valid for an arbitrary thickness third layer.

Consider wave motion in an isotropic and nonpiezoelectric material of density $\rho$ and with Lamé constants $\lambda$ and $\mu$.

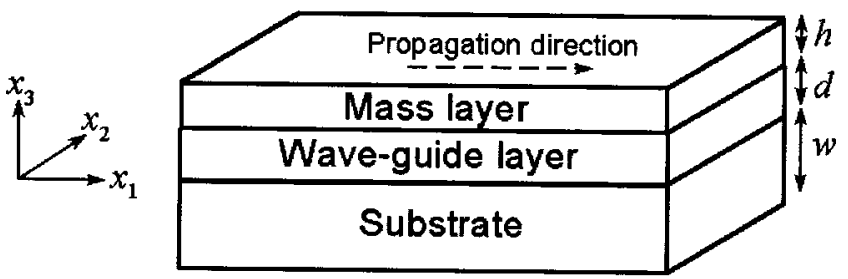

FIG. 1. Definition of axes and propagation direction for shear horizontally polarized waves in a three-layer system; the displacement is in the $x_{2}$ direction.

The displacements, $u_{j}$, are then described by the equation of motion $^{15}$

$$
\rho \frac{\partial^{2} u_{j}}{\partial t^{2}}=(\lambda+\mu) \frac{\partial S_{i i}}{\partial x_{j}}+\mu \underline{\nabla}^{2} u_{j}
$$

where the Einstein summation convention has been used and the strain tensor, $S_{i j}$, is defined as

$$
S_{i j}=\frac{1}{2}\left(\frac{\partial u_{i}}{\partial x_{j}}+\frac{\partial u_{j}}{\partial x_{i}}\right) \text {. }
$$

The boundary conditions on any solution require consideration of the stress tensor, $T_{i j}$, which can be written in the form

$$
T_{i j}=\lambda \delta_{i j} S_{k k}+2 \mu S_{i j}
$$

The upper surface of the substrate is taken to be in the $\left(x_{1}, x_{2}\right)$ plane and located at $x_{3}=0$ (Fig. 1). The solutions of the equation of motion are chosen to have a propagation along the $x_{1}$ axis with displacements in the $x_{2}$ direction of the sagittal plane $\left(x_{2}, x_{3}\right)$. They must also satisfy the boundary conditions on the displacements $\underline{u}$ and the $T_{i 3}$ component of the stress tensors. These must both be continuous at the interfaces between the substrate and guiding layer, and between the guiding layer and perturbing mass layer. The $T_{i 3}$ component of the stress tensors must also vanish at the free surfaces of the substrate and the perturbing mass layer at $x_{3}=-w$ and $x_{3}=(d+h)$, respectively.

In order to preserve the notational similarity with the Love wave problem, a solution for the equation of motion is sought by using displacements in the guiding layer, $\underline{u}_{l}$, the substrate, $\underline{u}_{s}$, and the perturbing mass layer, $\underline{u}_{p}$, of

$$
\begin{aligned}
& \underline{u}_{l}=(0,1,0)\left\lfloor A_{l} e^{-j T_{l} x_{3}}+B_{l} e^{j T_{l} x_{3}}\right\rfloor e^{j\left(\omega t-k_{1} x_{1}\right)}, \\
& \underline{u}_{s}=(0,1,0)\left\lfloor C_{s} e^{-T_{s} x_{3}}+D_{s} e^{T_{s} x_{3}}\right] e^{j\left(\omega t-k_{1} x_{1}\right)}, \\
& \underline{u}_{p}=(0,1,0)\left[E_{p} e^{-j T_{p} x_{3}}+F_{p} e^{j T_{p} x_{3}}\right] e^{j\left(\omega t-k_{1} x_{1}\right)},
\end{aligned}
$$

where $\omega$ is the angular frequency and the wave vector is $k_{1}$ $=(\omega / \nu)^{1 / 2}$ where $\nu$ is the phase speed of the solution. $A_{l}$, $B_{l}, C_{s}, D_{s}, E_{p}, F_{p}$, are constants determined by the boundary conditions. A traditional Love wave solution occurs when the substrate thickness $w \rightarrow \infty$, the shear speed of the substrate, $\nu_{s}=\left(\mu_{s} / \rho_{s}\right)^{1 / 2}$, is greater than the shear speed of the layer, $\nu_{l}=\left(\mu_{l} / \rho_{l}\right)^{1 / 2}$, and the wave vector $T_{s}$ is real, so that the substrate displacement, $\underline{u}_{s}$, decays with depth. A traditional SH-APM solution occurs when $w$ is finite, $d$ $\rightarrow 0$ and the wave vector $T_{s}$ is purely imaginary, so that the solution, $\underline{u}_{s}$, may take on a standing wave (resonant) form. 
In the more general case under consideration here, both $T_{l}$ and $T_{s}$ may be complex rather than real and no restriction to real is placed upon $T_{p}$. The use of the exponentials with a $j$ factor in Eq. (4) and without a $j$ factor in Eq. (5) is therefore purely to enable the similarity with the Love wave theory to be more readily noted. The choice of the exponential with a $j$ factor in Eq. (6) is to emphasize the notational similarity with the guiding layer. One particularly simple limit of the theory is to regard the perturbing mass layer as nothing more than an extension of the guiding layer itself, by setting the material properties to the same values.

Substituting Eq. (4) into the equation of motion describing the layers, i.e., Eq. (1) with the relevant layer parameters, gives the equations for the wave vectors $T_{s}, T_{l}$, and $T_{p}$ :

$$
\begin{aligned}
& T_{s}^{2}=\omega^{2}\left(\frac{1}{v^{2}}-\frac{1}{v_{s}^{2}}\right), \\
& T_{l}^{2}=\omega^{2}\left(\frac{1}{v_{l}^{2}}-\frac{1}{v^{2}}\right), \\
& T_{p}^{2}=\omega^{2}\left(\frac{1}{v_{p}^{2}}-\frac{1}{v^{2}}\right) .
\end{aligned}
$$

To completely specify the problem the boundary conditions are imposed and this defines the constants $A_{l}, B_{l}, C_{s}, D_{s}$, $E_{p}$, and $F_{p}$ in Eqs. (4)-(6). The first type of boundary condition is continuity of the displacements at the interfaces between the layers and these give

$$
\begin{aligned}
& A_{l}+B_{l}=C_{s}+D_{s}, \\
& \begin{array}{l}
A_{l} \exp \left(-j T_{l} d\right)+B_{l} \exp \left(j T_{l} d\right) \\
\quad=E_{p} \exp \left(-j T_{p} d\right)+F_{p} \exp \left(j T_{p} d\right) .
\end{array}
\end{aligned}
$$

The remaining conditions all relate to the $T_{i 3}$ component of the stress tensor, which for this system using the form of the solutions in Eqs. (4)-(6) can be written as

$$
T_{i 3}=\delta_{i 2} \mu\left(\frac{\partial u_{2}}{\partial x_{3}}\right) .
$$

The second type of boundary condition, continuity of $T_{i 3}$ at the substrate-layer interface, gives

$$
-A_{l}+B_{l}=j\left(C_{s}-D_{s}\right) \xi
$$

and

$$
\begin{aligned}
& -A_{l} \exp \left(-j T_{l} d\right)+B_{l} \exp \left(j T_{l} d\right) \\
& \quad=\left\lfloor-E_{p} \exp \left(-j T_{p} d\right)+F_{p} \exp \left(j T_{p} d\right)\right\rfloor \xi_{p},
\end{aligned}
$$

where $\xi$ and $\xi_{p}$ have been defined as

$$
\xi=\frac{\mu_{s} T_{s}}{\mu_{l} T_{l}}
$$

and

$$
\xi_{p}=\frac{\mu_{p} T_{p}}{\mu_{l} T_{l}} .
$$

The remaining two boundary conditions are vanishing of stress at the two free surfaces at $x_{3}=(d+h)$ and $x_{3}=-w$, and these give the equations

$$
E_{p} \exp \left[-j T_{p}(d+h)\right]-F_{p} \exp \left[j T_{p}(d+h)\right]=0
$$

and

$$
C_{s} \exp \left(T_{s} w\right)-B_{s} \exp \left(-T_{s} w\right)=0 .
$$

The six boundary conditions, Eqs. (10), (11), (13), (14), (17), and (18), define both a dispersion equation and the coefficients, $A_{l}, B_{l}, C_{s}, D_{s}, E_{p}$, and $F_{p}$, in the solutions for the displacements. After extensive algebraic manipulation we find the dispersion equation

$$
\begin{aligned}
\tan \left(T_{l} d\right)= & \xi \tanh \left(T_{s} w\right)-\xi_{p} \tan \left(T_{p} h\right) \\
& \times\left[1+\xi \tan \left(T_{l} d\right) \tanh \left(T_{s} w\right)\right] .
\end{aligned}
$$

The second term on the right-hand side of Eq. (19), which involves $\xi_{p} \tan \left(T_{p} h\right)$, is due to the presence of the third, perturbing, mass layer. Setting the thickness, $h$, of the perturbing mass layer to zero recovers the dispersion equation for the two-layer system of a substrate with a guiding layer. When the substrate thickness $w \rightarrow \infty$ with $T_{s}$ real, so that the $\tanh \left(T_{s} w\right) \rightarrow 1$, Eq. (19) gives the limit of a traditional Love wave perturbed by an arbitrary thickness perturbing mass layer. The layer guided $\mathrm{SH}-\mathrm{APMs}$ correspond to $T_{s}=j k_{s}$ where $k_{s}$ is real. ${ }^{17}$

\section{PERTURBATION THEORY}

When operated as a sensor, a Love wave device has a finite thickness wave-guiding layer; it is the finite thickness which is responsible for the high mass sensitivity. The presence of a finite thickness wave-guiding layer means that the wave speed for the Love waves is smaller than the substrate shear speed. Similarly, the wave speed for the layer guided SH-APMs are larger than the substrate shear speed. A third, thin perturbing, mass layer therefore acts about a particular operating point on the dispersion curve of the bare, twolayer, system defined by

$$
\tan \left(T_{l}^{0} d\right)=\xi^{0} \tanh \left(T_{s}^{0} w\right)
$$

where the superscripts on $T_{l}^{0}$ and $T_{s}^{0}$ indicate the wave vectors in Eqs. (7)-(9) are given by a solution to Eq. (20) for $\nu=\nu_{0} \neq v_{s}$; the superscript on $\xi^{0}$ indicates that $T_{l}^{0}$ and $T_{s}^{0}$ are used in Eq. (15). The solutions for this system have been discussed in detail in a previous report; ${ }^{17}$ it should be noted that the limit of a vanishing wave-guiding layer has to be handled carefully as this involves the conversion from layer guided acoustic plate modes with an imaginary wave vector to Love waves with a real wave vector.

Consider a perturbing third mass layer of thickness, $h$ $=\Delta h$. This perturbation of the two-layer system will result in a decrease in the phase speed of the mode, irrespective of whether that mode is a Love wave or a layer guided SHAPM. The perturbation will cause changes in the phase speeds and the wave vectors of the substrate and guiding layers and we can therefore write $T_{l}^{0} \rightarrow T_{l}^{0}+\Delta T_{l}, T_{s}^{0} \rightarrow T_{s}^{0}$ $+\Delta T_{s}$ and $\xi^{0} \rightarrow \xi^{0}+\Delta \xi$ where the superscript zero indicates the values of the quantities when $\Delta h=0$ [i.e., solutions of Eq. (20)]. The left-hand side of the three-layer dispersion equation [Eq. (19)] can then be written, 


$$
\tan \left(T_{l} d\right)=\frac{\tan \left(T_{l}^{0} d\right)+\tan \left(\Delta T_{l} d\right)}{1-\tanh \left(T_{l}^{0} d\right) \tan \left(\Delta T_{l} d\right)} .
$$

The first term on the right-hand side of the three-layer dispersion equation [Eq. (19)] can be written

$$
\xi \tanh \left(T_{s} w\right)=(\xi+\Delta \xi)\left[\frac{\tanh \left(T_{s}^{0} w\right)+\tanh \left(\Delta T_{s} w\right)}{1+\tanh \left(T_{s}^{0} w\right) \tanh \left(\Delta T_{s} w\right)}\right]
$$

and the second term can be written to first order as

$$
\begin{aligned}
& \xi_{p} \tan \left(T_{p} h\right)\left[1+\xi \tan \left(T_{l} d\right) \tanh \left(T_{s} \bar{w}\right)\right] \\
& \quad \approx \xi_{p}^{0} T_{p}^{0} \Delta h\left\lfloor 1+\xi^{0} \tan \left(T_{l}^{0} d\right) \tanh \left(T_{s}^{0} w\right)\right\rfloor .
\end{aligned}
$$

The expansion in $\Delta \xi$ can be written in terms of $\Delta T_{l}$ and $\Delta T_{s}$ using Eq. (15) and both of these quantities can be related to the change, $\Delta \nu$, in the wave speed, $\nu_{0}$, using Eqs. (7) and (8). Performing these manipulations and grouping terms, we find that Eq. (19) gives the perturbation formula

$$
\frac{\Delta \nu}{\nu_{0}} \approx\left(1-\frac{\nu_{p}^{2}}{\nu_{0}^{2}}\right) \rho_{p} g\left(\omega, \nu_{s}, \rho_{l}, \nu_{l}, w, d\right) \Delta h,
$$

where the function $g$, is defined as

$$
g\left(\omega, \nu_{s}, \rho_{l}, \nu_{l}, w, d\right)=\frac{\frac{\omega^{2}}{\rho_{l} T_{l}^{0} \nu_{l}^{2}}\left[1+\tan ^{2}\left(T_{l}^{0} d\right)\right]}{\frac{T_{l}^{0} d}{\left(\frac{\nu_{0}^{2}}{\nu_{l}^{2}}-1\right)}\left[1+\tan ^{2}\left(T_{l}^{0} d\right)\right]-\frac{T_{s}^{0} w}{\left(1-\frac{\nu_{0}^{2}}{\nu_{s}^{2}}\right)}\left[1-\tanh ^{2}\left(T_{l}^{0} w\right)\right]+\tan \left(T_{l}^{0} d\right)\left[\frac{1}{\left(\frac{\nu_{0}^{2}}{\nu_{l}^{2}}-1\right)}-\frac{1}{\left(1-\frac{\nu_{0}^{2}}{\nu_{s}^{2}}\right)}\right]}
$$

and depends only on the operating frequency and properties of the substrate and wave-guiding layer. These formulas are only valid for perturbations about an operating point on the two-layer dispersion curve that satisfies $\nu_{0} \neq \nu_{s}$ and $\nu_{0}$ $\neq \nu_{l}$; a discussion of the difficulties of perturbing the two layer dispersion curve about the start of a Love wave mode, or the so called $n=0 \mathrm{SH}-\mathrm{APM}$ mode, has been given in Ref. 17. It should be noted that due to the frequency dependence of $T_{l}^{0}$ and $T_{s}^{0}$ in the function, $g$, Eq. (24) does not, in general, predict a frequency-squared dependence for the fractional shift in phase speed. The function $g$ determines the mass sensitivity of the sensor device. The structure of the formula in Eq. (24) is entirely consistent with the result given by Auld ${ }^{18}$

$$
\frac{\Delta \nu}{\nu_{0}} \approx-\frac{\nu_{0}}{4}\left(1-\frac{\nu_{p}^{2}}{\nu_{0}^{2}}\right) \rho_{p}\left|U_{2}\right|^{2} \Delta h,
$$

where $U_{2}$ is the normalized particle velocity displacement at the surface. The combination, $\rho_{p} \Delta h$, of the density and thickness of the perturbing mass layer gives the mass per unit surface area, $\Delta m$, and we can therefore rewrite Eq. (24) as

$$
\frac{\Delta \nu}{\nu_{0}} \approx\left(1-\frac{\nu_{p}^{2}}{\nu_{0}^{2}}\right) g\left(\omega, \nu_{s}, \rho_{l}, \nu_{l}, w, d\right) \Delta m .
$$

\section{MASS SENSITIVITY FROM THE DEVICE DISPERSION CURVE}

Experimentally, the significance of Eqs. (24) and (25) is that if we can determine the sensitivity function, $g$, for any perturbing layer, then it is the same function for any other perturbing mass layer. Now consider a two-layer system and imagine creating a thin third layer of the same material as the wave-guiding layer. Writing $x=d$ and $\Delta h=\Delta x$, Eq. (24) becomes

$$
\frac{\Delta \nu}{\nu_{0}} \approx g\left(\omega, \nu_{s}, \rho_{l}, \nu_{l}, w, d\right)\left(1-\frac{\nu_{l}^{2}}{\nu_{0}^{2}}\right) \rho_{l} \Delta x,
$$

where the subscript zero indicates the value of the phase speed at a thickness $x=d$. Making the third layer infinitesimally thin we may write the function, $g$, as

$$
g\left(\omega, \nu_{s}, \rho_{l}, \nu_{l}, w, d\right)=\frac{1}{\nu_{0} \rho_{l}\left(1-\nu_{l}^{2} / \nu_{0}^{2}\right)}\left(\frac{d \nu}{d x}\right)_{x=d} .
$$

Thus, the sensitivity function involves the slope of the phase velocity dispersion curve with guiding layer thickness at the guiding layer thickness operating point. Using Eq. (29) we may simplify Eq. (24) to

$$
\frac{\Delta \nu}{\nu_{0}} \approx \frac{\rho_{p}}{\rho_{l}}\left[\frac{1-\nu_{p}^{2} / \nu_{0}^{2}}{1-\nu_{l}^{2} / \nu_{0}^{2}}\right] \frac{1}{\nu_{0}}\left(\frac{d \nu}{d x}\right)_{x=d} \Delta h
$$

and this may be further simplified if $\nu_{p}^{2} \ll \nu_{0}^{2}$ and $\nu_{l}^{2} \ll \nu_{0}^{2}$. Equation (30) can be rewritten using the perturbing mass $\Delta m=\rho_{p} \Delta h$. Equation (30) should be of particular use in developing wave guide-based acoustic wave sensors, because it enables the mass sensitivity of a prospective device to be assessed directly from the dispersion curve. Moreover, this dispersion curve can be determined either numerically or from experimentation by systematically increasing the thickness of the wave guide layer. Whilst arguments based on perturbation theory have been used in deriving Eq. (30), the formula itself is for a perturbation on top of a wave guide layer of arbitrary thickness rather than of a vanishing thickness. Defining a mass sensitivity function, $S_{m}$, we can write 


$$
S_{m}=\lim _{\Delta m \rightarrow 0} \frac{1}{\Delta m}\left(\frac{\Delta \nu}{\nu_{0}}\right)=\frac{1}{\rho_{l}}\left[\frac{1-\nu_{p}^{2} / \nu_{0}^{2}}{1-\nu_{l}^{2} / \nu_{0}^{2}}\right] \frac{1}{\nu_{0}}\left(\frac{d \nu}{d x}\right)_{x=d}
$$

or

$$
S_{m}=\frac{1}{\rho_{l}}\left[\frac{1-\nu_{p}^{2} / \nu_{0}^{2}}{1-\nu_{l}^{2} / \nu_{0}^{2}}\right]\left(\frac{d \log _{e} \nu}{d x}\right)_{x=d},
$$

where the mass sensitivity function, $S_{m}$, is in units of $\mathrm{m}^{2} \mathrm{~kg}^{-1}$. Thus, the mass sensitivity of a Love wave or a layer-guided shear horizontally polarized acoustic plate mode device can be determined numerically from the dispersion curve. Whilst a relationship between the maximum slope in a dispersion curve and the maximum mass sensitivity for surface acoustic wave sensors has been remarked upon by some researchers, ${ }^{19}$ Eq. (32) gives it an explicit theoretical basis for Love waves and layer guided SHAPMs.

It is interesting to note that an often drawn conclusion from Auld's result, Eq. (26), is that the device sensitivity increases as $U_{2}$ at the sensing surface increases and that this corresponds to a trapping of the acoustic energy to the surface. If we assume $\nu_{p} \approx \nu_{l}$, then Eq. (32) only involves the slope of the logarithm of the dispersion curve and, for Love waves, this slope is a maximum at the transition from a wave dominantly in the substrate to one dominantly in the wave guide layer. At greater wave guide thicknesses the Love wave mode is increasingly localized in the wave guide layer, compared to the substrate, but this leads to lower mass sensitivity rather than higher mass sensitivity; this conclusion applies only when a particular Love wave mode is maintained throughout the increase in wave guide thickness. Thus, to create a highly mass sensitive device the wave guide layer thickness should be chosen such that the wave is close to a transition between the two intrinsic wave speeds $\nu_{s}$ and $\nu_{l}$. The wave guide layer and substrate materials should also be chosen to obtain a sharp transition in the dispersion curve; for high mass sensitivity the aim is not to fully confine the wave to the guiding layer, but to place the operating point on the transition point of the dispersion curve. This change of emphasis in interpretation away from focusing on $U_{2}$ is important in understanding the potential of the layer guided SH-APM modes. The layer guided SH-APM modes have a wave speed larger than the substrate speed, $\nu_{s}$, but can still possess a sharp transition (with guiding layer thickness) between two intrinsic plate mode speeds. Moreover, by arranging the substrate thickness appropriately, the two plate mode speeds involved in the transition can be well-separated in value so that the slope in the dispersion curve can be large and the mass sensitivity can be high. In fact, the Love wave case involves a transition between $\nu_{s}$ and $\nu_{l}$, and so the change in speed due to the transition cannot be larger than $\nu_{s}$ whereas no such restriction occurs for a layer guided SHAPM sensor. It may therefore be possible to create a layer guided SH-APM sensor using the same guiding layer and substrate materials as a Love wave device, but with higher mass sensitivity and with the advantage of being able to excite the mode using transducers on the opposite side to the sensing surface. This possibility is further investigated in Sec. VI using numerical calculations for the dispersion curves.

\section{MASS SENSITIVITY AND FREQUENCY DEPENDENCE}

In a two-layer system with a finite thickness substrate and finite thickness wave-guiding layer, the frequency enters the calculation of the wave speed, $\nu_{0}$, through the two dimensionless combinations $d / \lambda_{l}=d f / \nu_{l}$ and $w / \lambda_{s}=w f / \nu_{s}$. When the substrate is infinitely thick the layer guided plate modes are no longer possible and only Love waves can exist. Moreover, on such an infinite thickness substrate the phase speed for a Love wave depends on frequency only through the dimensionless combination of $z=d / \lambda_{l}=d f / \nu_{l}$, so that a change in guiding-layer thickness, $d$, is equivalent to a change in operating frequency, $f$. Thus, Eq. (32) [or Eq. (31)] can be used to assess the change in sensitivity that will occur through a change in operating frequency, for a given mass perturbation, $\Delta m$, on a particular device. The dispersion curve can be plotted using the dimensionless variable, $z$, and the slope on this dispersion curve can be related to the slope in the dispersion curve when plotted against guiding layer thickness

$$
\left(\frac{d \nu}{d x}\right)_{x=x_{0}}=\frac{f_{0}}{\nu_{l}}\left(\frac{d \nu}{d z}\right)_{z=z_{0}},
$$

where the subscript o implies the values of the various quantities at the operating point of the dispersion curve. The mass sensitivity function, Eq. (32), then becomes

$$
S_{m}=\frac{1}{\rho_{l}}\left[\frac{1-\nu_{p}^{2} / \nu_{0}^{2}}{1-\nu_{l}^{2} / \nu_{0}^{2}}\right] \frac{f_{0}}{\nu_{l}}\left(\frac{d \log _{e} \nu}{d z}\right)_{z=z_{0}} .
$$

One immediate consequence of Eq. (34) is that for a given Love wave mode the peak sensitivity (maximum value of $S_{m}$ ) is directly proportional to frequency. This is because any frequency increase can be accompanied by a corresponding reduction in the value of the guiding layer thickness so as to keep the value of $z$ constant at the appropriate operating point for the maximum sensitivity of the dispersion curve; neither the maximum value of the differential of the log, nor $\nu_{0}$, at this value of $z$ change with frequency. The shift in phase velocity (at fixed operating frequency) due to sensed mass for a Love wave device will scale with the frequency, provided the guiding layer thickness has been chosen to obtain maximum sensitivity for that operating frequency and the same Love wave mode is used at each operating frequency. If the guiding layer thickness is different to the optimal one for maximum mass sensitivity then the gain in phase velocity sensitivity would not scale with $f$. Also, if a frequency change is made that takes a device from one Love wave mode to another then the maximum gain in sensitivity would be less than the $f$ factor because the peak values of the differential of the log term in Eq. (34) will be different. Love wave devices are dispersive so that frequency shift, which is measured in oscillator configurations, due to added mass is given by $\Delta f / f=\left(\nu_{g} / \nu\right)(\Delta \nu / \nu)$, where $\nu_{g}$ is the group ve- 


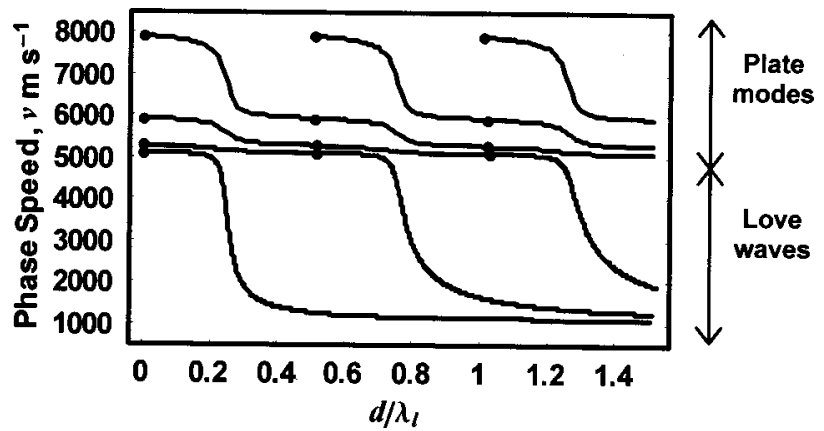

FIG. 2. Calculated dispersion curves as a function of normalized guiding layer thickness $\left(d / \lambda_{l}=d f / v_{l}\right)$ for the two-layer system of a substrate and a wave-guiding layer. The multiple modes of Love waves have $v<v_{s}$ and the associated acoustic plate modes have $v>v_{s}$. The solid circle symbols indicate the analytical result for the start of each mode.

locity. The ratio of group to phase velocity varies along the dispersion curve and the translation of mass sensitivity from the phase speed definition of Eq. (31) to $\Delta f / f$ therefore requires care.

In previous work, we have reported that Love wave devices can be designed with transducers that operate at harmonic frequencies ${ }^{20}$ (see also Weiss et al. ${ }^{21}$ ). This enables a single device with a given guiding layer to be "hopped" from one operating frequency to another during the course of a single sensing experiment. Thus, it is of particular interest to know the change in mass sensitivity of a Love wave device that arises by changing frequency, whilst keeping the wave guide thickness constant, so that the operating point on the dispersion curve is altered. From Eq. (34), the mass sensitivity, $S_{2}$, at a frequency $f_{2}$ compared to the mass sensitivity, $S_{1}$, at a frequency $f_{1}$, is given by

$S_{2}=\left(\frac{1-\nu_{p}^{2} / \nu_{2}^{2}}{1-\nu_{p}^{2} / \nu_{1}^{2}}\right)\left(\frac{1-\nu_{l}^{2} / \nu_{1}^{2}}{1-\nu_{l}^{2} / \nu_{2}^{2}}\right)\left(\frac{f_{2}}{f_{1}}\right)\left[\frac{\left(\frac{d \log _{e} \nu}{d z}\right)_{z=z_{2}}}{\left(\frac{d \log _{e} v}{d z}\right)_{z=z_{1}}}\right] S_{1}$.

It should be noted that Eq. (35) is valid whether or not the change in frequency leads to a Love wave of the same mode. In a harmonic type device design the frequency change would typically be a doubling or trebling and could therefore involve a change of Love wave mode.

\section{NUMERICAL SOLUTIONS AND DISCUSSION}

The numerical solution of the dispersion equation for the two-layer problem of a finite thickness substrate covered by a wave-guiding layer has previously been considered. ${ }^{16,17}$ In general, for any given guiding layer thickness the phase speed is multiple valued with both multiple layer guided SH-APM modes and multiple Love wave modes. Solutions with phase speeds greater than the substrate shear velocity, $\nu_{s}$, are layer-guided SH-APMs and solutions with phase speeds less than the substrate shear velocity, $\nu_{s}$, are generalizations of Love waves to a finite thickness substrate. Figure 2 shows the dispersion curve diagram for an operating

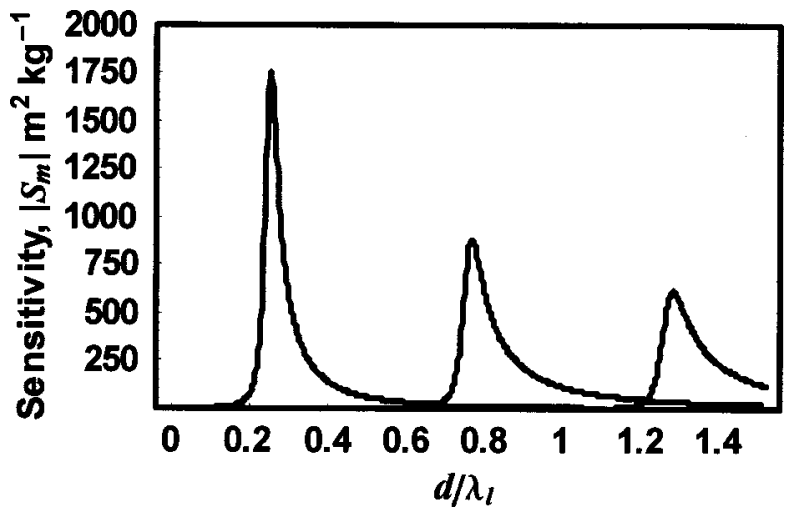

FIG. 3. Mass sensitivity, $\left|S_{m}\right|$, in $\mathrm{m}^{2} \mathrm{~kg}^{-1}$ for the three Love wave modes shown in Fig. $2 ; v_{p}=v_{l}$ has been used in the calculation using Eq. (34).

frequency of $f=100 \mathrm{MHz}$ on a substrate of thickness $w$ $=100 \mu \mathrm{m}$ and with density and substrate speed typical of quartz $\left(\rho_{s}=2655 \mathrm{~kg} \mathrm{~m}^{-3}\right.$ and $\left.\nu_{s}=5100 \mathrm{~ms}^{-1}\right)$. The wave guide layer parameters are $\rho_{l}=1000 \mathrm{~kg} \mathrm{~m}^{-3}$ and $\nu_{l}$ $=1100 \mathrm{~ms}^{-1}$ and correspond to poly(methylmethacrylate) (PMMA). The horizontal axis has been plotted using a dimensionless parameter of the wave-guiding layer thickness scaled by $\lambda_{l}=\nu_{l} / f$. The solid circles on the curves indicate thicknesses at which solutions have been determined analytically as well as numerically. In the simplest interpretation of mass sensitivity Eq. (34), suggests that the maximum sensitivity occurs at the maximum slope of the curves. Figure 3 shows the modulus of the mass sensitivity, $\left|S_{m}\right|$, calculated from Eq. (34) for the first three Love wave modes in Fig. 2. The corresponding curves for the layer-guided SH-APM modes are shown in Fig. 4. As anticipated the maximum sensitivity occurs on the backslope of each mode in Fig. 2 which, for the parameter values used for the calculations, is at a guiding layer thickness of $d \sim(2 n+1) \lambda_{l} / 4$ where $n$ is an integer.

The numerical calculations of the mass sensitivity of the layer-guided SH-APM modes can be compared to the analytical results for a bare SH-APM device perturbed by a thin mass layer. Martin et al. ${ }^{7,22}$ give the formula $S_{m}$ $=-1 /\left(\rho_{s} w\right)$ for the $n>0$ modes of a SH-APM device and one-half this value for the $n=0$ mode. However, in our pre-

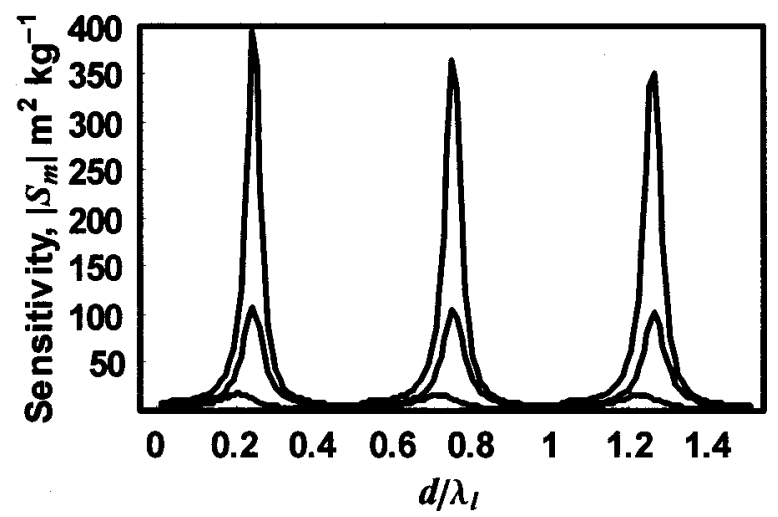

FIG. 4. Mass sensitivity, $\left|S_{m}\right|$, in $\mathrm{m}^{2} \mathrm{~kg}^{-1}$ for the layer guided SH-APM modes associated with the three Love wave modes shown in Fig. $2 ; v_{p}$ $=v_{l}$ has been used in the calculation using Eq. (34). 
vious work we demonstrated that under mass loading the $n$ $=0$ APM degenerates into a Love wave and the previously quoted result for the $n=0$ mode does not therefore apply. ${ }^{17}$ We also derived the following more general result for the perturbation of the $n>0$ modes of a bare SH-APM device

$$
S_{m}=\left(\frac{\nu_{l}^{2}}{\nu_{s}^{2}}\right)\left(1-\frac{\nu_{m}^{2}}{\nu_{l}^{2}}\right)\left(\frac{1}{\rho_{s} w}\right),
$$

where $\nu_{m}$ is the unperturbed SH-APM mode speed of the bare device. For the calculations in Figs. 2 and 4 the SHAPM modes have speeds of 5274.36, 5929.03, and $7918.88 \mathrm{~m} \mathrm{~s}^{-1}$ compared to the substrate speed of $5100 \mathrm{~m} \mathrm{~s}^{-1}$. The extra prefactors in Eq. (36) are therefore important because $\nu_{m}$ is not approximately equal to the substrate shear speed, $\nu_{s}$. Evaluating Eq. (36) gives mass sensitivities of $S_{m}=3.85,4.92$, and $8.91 \mathrm{~m}^{2} \mathrm{~kg}^{-1}$, respectively, for the three $n>0 \mathrm{SH}-\mathrm{APM}$ modes and these are in agreement with the numerical values in Fig. 4 in the limit $d \rightarrow 0$. From Fig. 4 it can be seen that the effect of the guiding layer on the SH-APM device is to dramatically increase the mass sensitivity by more than an order of magnitude. The greatest gain in mass sensitivity is with the highest order SH-APM mode. For the calculations in Fig. 2 the mass sensitivity of the layer-guided SH-APMs becomes comparable, to within an order of magnitude, of the mass sensitivity of the Love wave modes.

Physically it is possible to understand the high mass sensitivity that can be obtained in layer guided acoustic wave systems by considering the change in the displacements as the guiding layer thickness increases. Beginning with the first Love wave mode and increasing the wave-guiding layer thickness from zero, takes the displacement pattern from a plane wave in the substrate and layer, to one with virtually no displacement in the substrate, but a quarter-wavelength type pattern in the guiding layer. ${ }^{17}$ Further increases in guiding layer thickness will further confine the displacement to the wave guide layer, but do not then correspond to higher sensitivity. This increase in the wave guide layer thickness corresponds to taking the Love wave speed from a value equal to the substrate speed, $\nu_{s}$, to a value close to the layer speed, $\nu_{l}$. Further increases in the wave guide layer thickness do not significantly alter the wave speed of this Love wave mode and so give poor mass sensitivity. However, increasing the thickness of the wave-guiding layer does eventually gives rise to higher order Love wave modes which go through similar changes in the wave speed (i.e., from $\nu_{s}$ to $\nu_{l}$ ). In the case of the second Love wave mode the displacement pattern begins as a plane wave in the substrate and a half-wavelength type pattern in the guiding layer. This pattern evolves until it becomes one with virtually no displacement in the substrate, but with a three quarter-wavelength type pattern in the guiding layer. ${ }^{17}$ In the Love wave mode case, the maximum mass sensitivity occurs at the point of transition of the Love wave from having properties similar to those of a shear wave in the substrate to one with properties similar to those of a shear wave in the layer. In a similar manner, the layer-guided SH-APM modes change character from one plate resonance to the next lower order plate resonance as the wave guide layer thickness increases. For ex- ample, a transition from a plate mode with a $3 / 2$ wavelength pattern in the substrate to one with a $1 / 2$ wavelength pattern in the substrate. The maximum mass sensitivity is when a device is operated with a wave-guiding layer possessing a thickness chosen so that the displacement pattern is at one of these points of transition. This corresponds to the point on the dispersion curve where the phase speed changes most rapidly with guiding layer thickness.

In acoustic wave sensor research it is often quoted that Love wave sensors have a higher sensitivity than SH-APM sensors whilst SH-APM sensors have the advantage that the transducers can be on the opposite face to that used for sensing. In liquids, this latter property can be a significant advantage. The comparison that leads to the belief that Love wave sensors are more sensitive than SH-APM devices does not account for the dispersion curves of the layer-guided SHAPMs shown in Fig. 2. The usual comparison is between a bare $\mathrm{SH}-\mathrm{APM}$ device, which therefore corresponds on Fig. 2 to the slope of the dispersion curve with a zero thickness wave-guiding layer, and a Love wave device chosen to have a wave-guiding layer thickness corresponding to the maximum slope in the dispersion curve. Clearly, if a waveguiding layer is used for the SH-APM device, then an operating point corresponding to the maximum slope on the dispersion curve can be chosen and the difference in mass sensitivities is much less. In the case of the calculations in Fig. 2 the maximum sensitivity of the highest mode layer guided SH-APM is within a factor of 5 of that of the Love wave. However, in general we would argue that a layerguided SH-APM device cannot only be of comparable sensitivity, but may in fact be more sensitive than a Love wave on a given substrate. This is because the maximum change in wave speed for the Love wave is $\left(\nu_{s}-\nu_{l}\right)$ and this occurs over a small range of guiding layer thickness centred around a thickness of $(2 n+1) \lambda_{l} / 4$. In comparison, the substrate thickness, $w$, can be chosen such that the change in speed for the highest order layer-guided SH-APM can be far greater than the difference $\left(\nu_{s}-\nu_{l}\right)$; again this change will occur over a small range of guiding layer thickness centred around a thickness of $(2 n+1) \lambda_{l} / 4$. To further illustrate this idea, we have numerically calculated the mass sensitivity using the same materials and operating frequency as in Figs. 2 and 3, but with the substrate thickness modified to $77 \mu \mathrm{m}$. In this case the initial mode speeds for the SH-APMs without a guiding layer are 5405,6807 , and $44825 \mathrm{~m} \mathrm{~s}^{-1}$. This particular choice of substrate thickness creates a large difference in speed between the top two modes and we therefore anticipate that introducing a guiding layer will provide increased mass sensitivity compared to the device on the $100 \mu \mathrm{m}$ substrate. The numerical comparison between the mass sensitivity of the Love wave and the associated highest order layer-guided SH-APM for the first three Love wave modes is shown in Fig. 5. The layer-guided SH-APMs are shown by the dotted curves and the Love waves by the solid curves. The mass sensitivity of the Love waves has not changed significantly compared to Fig. 3, but that of the layer guided SH-APMs has increased and now exceeds the mass sensitivity of the Love waves. It is also evident that progressing through the sequence of Love wave modes, the peak mass sensitivity of 


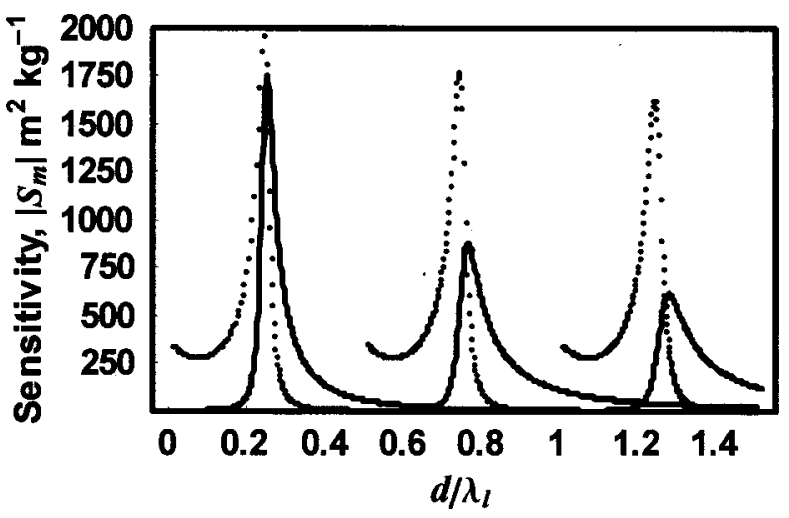

FIG. 5. Comparison of the mass sensitivity, $\left|S_{m}\right|$, in $\mathrm{m}^{2} \mathrm{~kg}^{-1}$ for the three highest layer guided SH-APM modes (dotted curves) associated with the first three Love wave modes (solid curves) using a reduced substrate thickness of $w=77 \mu \mathrm{m}$. All other parameters are the same as in Fig. 4 .

the highest layer-guided SH-APM mode associated with each higher order Love wave mode suffer less of a drop than that of the higher order Love wave modes (i.e., the sequence of peak values of the dotted curves in Fig. 5 decreases less rapidly than that of the solid curves in Fig. 5). The particular choice for the substrate thickness used in Fig. 5 was extreme and fabricating devices to obtain this sensitivity may prove difficult due to the high phase speed of the mode. However, choosing substrate thicknesses to give a top SH-APM with a speed of the order of $10000 \mathrm{~m} \mathrm{~s}^{-1}$ would still give mass sensitivities comparable to Love wave devices. Thus, by using a layer-guided SH-APM it should be possible to reconcile the requirements for high mass sensitivity with that of operating a device with transducers on the opposite face to the sensing surface.

In sensors using quartz crystal microbalances and surface acoustic waves, layer guided or otherwise, higher frequency is usually believed to result in higher sensitivity. Some aspects of this possible frequency enhancement of sensitivity for Love waves on an infinite substrate have been discussed in Sec. V where it was shown that the peak sensitivity for a given Love wave mode can scale with frequency, but that this requires a corresponding reduction in guiding layer thickness. Figures 2 and 3 emphasize that increasing frequency with a given device of fixed guiding layer thickness below the peak in sensitivity will increase the value of $z$ and so increase sensitivity. Since the dispersion curve for a given mode is not linear, the frequency gain for such a device operating away from the peak sensitivity for the mode will not be linear with frequency. To further understand the frequency dependence, imagine a Love wave on an infinite thickness substrate and with the guiding layer thickness optimized to give maximum sensitivity for the first Love wave mode. The dispersion curve will look similar to the first Love wave mode in Fig. 2 and the operating point will be at around $z=d / \lambda_{l} \sim \frac{1}{4}$ where $\lambda_{l}=\nu_{l} / f$. As shown in Sec. V, on an infinite thickness substrate the frequency only enters the calculation of the dispersion curve in combination with the guiding layer thickness through $z=d / \lambda_{l}$. Thus, if we keep the guiding layer thickness, $d$, constant and increase the frequency we will move the operating point along the horizon-

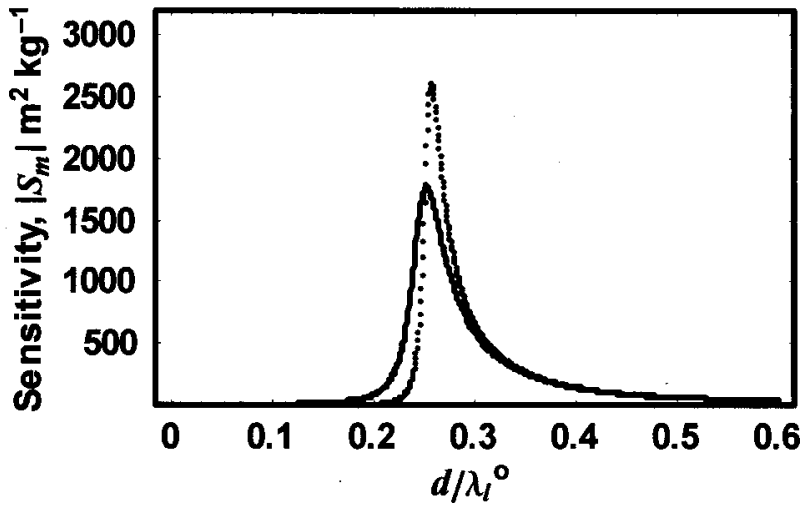

FIG. 6. Comparison of the sensitivity, $\left|S_{m}\right|$, in $\mathrm{m}^{2} \mathrm{~kg}^{-1}$ for the mode 1 Love wave and the corresponding mass sensitivity for the mode 2 Love wave obtained by increasing the frequency by a factor of 3 whilst maintaining the guiding layer thickness constant; the substrate is assumed infinite thickness. The horizontal axis for both data sets has been plotted using the original coordinate before the frequency was increased.

tal axis of the dispersion curve. The sensitivity at the new operating point is related to the slope of the dispersion curve and the frequency through Eq. (34) [or Eq. (35)]. An immediate conclusion from this viewpoint is that approximately doubling the frequency will lead to either the same Love wave mode, but with much lower sensitivity, or the next higher Love wave mode, again with a much lower sensitivity. Alternatively, trebling the frequency will lead to either the same mode, but then with low sensitivity, or a point close to, but not exactly at, the optimum on the dispersion curve for the second Love wave mode $\left(z=d / \lambda_{l} \sim 3 / 4\right)$. These conclusions are relevant experimentally as it is possible to design Love wave devices capable of hopping between several frequencies during the course of a sensor experiment by fabricating specific patterns of the interdigital transducers used to excite Love waves. ${ }^{20}$

Figure 6 illustrates the effect of altering the initial frequency, $f_{0}$, by a factor of three to $3 f_{0}$ so that the operating point moves from the first to the second Love wave mode. The horizontal axis data for the higher frequency has been plotted using the original coordinate $z=d f_{0} / \nu_{l}$ so that a direct comparison of the sensitivities at the two frequencies can be made by reading at the same value on the horizontal axis. Apart from the substrate thickness, which has been set to infinite, the parameters in the calculation are the same as in Fig. 2. A choice of $\nu_{p}=\nu_{l}$ has again been used to help the comparison and physically this corresponds to mass sensitivity towards the same material as the guiding layer. Importantly, if the guiding layer thickness has been selected optimally to give the maximum sensitivity of the first Love wave mode at the operating frequency, $f_{0}$, then increasing the frequency by a factor of 3 does not result in a significant change in sensitivity. However, it is more likely experimentally for a wave-guiding layer thickness to be selected that is not quite optimal for the maximum mass sensitivity in the first Love wave mode. In this situation frequency hopping by trebling the frequency could result in either a greater or smaller mass sensitivity. It is possible to align the peak sensitivities between two frequencies that use mode 1 and mode 2 Love 
waves for the layer and substrate materials used in the calculation of Fig. 6 by changing the frequency by a factor of around 3.04; the ratio of peak sensitivites is then 1.51. Similarly, frequency hopping by a factor of 5.08 will move the peak sensitivity of mode 1 to the peak sensitivity of mode 3 and give a relative increase in sensitivity of around 1.77. A change in frequency by a factor of 3.04 by frequency hopping would not be difficult experimentally as typical transducers have bandwidths of a few percent. Aligning the peak sensitivities of two modes when frequency hopping still does not give the factor of $f$ gain in sensitivity that could be expected by using two devices with their wave guide layer thickness optimized for maximum sensitivity for Love wave mode 1 at frequencies of $f$ and $3.04 f$, respectively. This is because the peak value of the $d \log _{e} z / d z$ in Eq. (34) is less at the higher mode. It should also be emphasized that the comparison made in this section is for the sensitivity function, which is the fractional change in phase speed rather than the absolute change in phase speed.

A final point of interest to current experimental work that arises from Fig. 3, is the manner in which the mass sensitivity for Love waves changes with thick guiding layers. Often, polymer materials, such as PMMA, are chosen as wave guide layers even though such materials can have significant attenuation for shear wave propagation. Therefore, as such a wave guide layer becomes thicker it is expected that the insertion loss of the Love wave device should significantly increase. Experimentally, this can be preceded by an initial improvement in insertion loss, if the substrate is chosen to use a surface skimming bulk wave (SSBW) rather than a pure SH-SAW mode. Thus, an optimized sensor usually involves choosing a wave-guiding layer thickness as a compromise between the maximum phase velocity sensitivity (i.e., maximum slope in the dispersion curve) whilst not placing the operating point so far down the dispersion curve for the first Love wave mode that the insertion loss is intolerable. It is easy to believe that once a guiding layer thickness causes a large insertion loss, no reasonable sensor can be obtained by further increases in the guiding layer thickness. This is not, however, the case. Experimentally, it is known that a relatively strong Love wave, with a relatively acceptable insertion loss, can reoccur periodically, as the guiding layer thickness is further increased; ${ }^{23}$ these correspond to higher order Love wave modes. In our experimental results using a polymer photoresist wave guide layer on a SSBW device, we have seen more than seven such modes. Figure 3 shows that the mass sensitivity of the higher order modes is, to within a factor of $2-3$, comparable to the first Love wave mode. Physically, the start of each Love wave mode corresponds to a displacement supported in the substrate; for a finite thickness substrate these involve antinodes at each of the free surfaces. This substrate displacement supports the wave despite the intrinsic loss of the polymer and it is only as the polymer thickness is further increased, from that corresponding to the start of the mode, that the substrate motion is reduced and the wave more fully localized into the guiding layer. Once the localization occurs, the damping of the polymer becomes fully effective, the insertion loss rises and the Love wave is damped. We would expect the layer- guided SH-APM modes to have a similar behavior for the insertion loss. An important conclusion from this interpretation is that it should be possible to use relatively thick waveguiding layers with these types of acoustic wave sensors (Love waves and layer-guided SH-APMs) without completely sacrificing mass sensitivity. This should widen the range of wave guide materials that can be used with layerguided acoustic wave sensors. Another consequence of the relationship between insertion loss and localization of the Love wave is that frequency hopping by a factor of 3 for a device optimized for mass sensitivity in the first mode will cause the operating point to move to the next mode rather than a lower point on the same Love wave mode.

In this report, all derivations and calculations have referred to a third layer composed of rigid mass. However, the method adopted could be extended to a third layer that is either a liquid or a viscoelastic material by, for example, introducing a Maxwell model with a relaxation time. Indeed, we would anticipate that layer guided SH-APM sensors would benefit from the same enhancement of sensitivity over SH-APM modes when being used to determine liquid properties, such as a density-viscosity product, or the shear modulus of a polymer.

\section{CONCLUSION}

The concept of mass sensing using Love waves and layer-guided shear horizontal polarized acoustic plate modes on finite thickness substrates has been developed using a dispersion equation for a three layer system. Formulas for the mass sensitivity have been derived and the relative sensitivity of Love wave and layer-guided SH-APM modes considered. Numerical calculations of the formulas show that the introduction of the guiding layer onto a SH-APM sensor can increase the mass sensitivity by several orders of magnitude and may even result in mass sensitivities exceeding those of Love wave devices. It is predicted that layer-guided SH-APM sensors having comparable or better sensitivity to Love wave sensors, but having the advantage of transducers on the opposite face to the sensing surface should be possible. The relationship between mass sensitivity and the slope of the numerically or experimentally determined dispersion curve has been considered. The effect of changing the operating frequency of a given Love wave device has also been considered on the basis of the slope of the dispersion curve. It has been shown that peak sensitivity scales linearly with frequency provided the Love wave mode does not change, but that hopping the frequency so that the operating device changes Love wave modes will give a lower increase in obtainable peak sensitivity. The mass sensitivity of sensor devices with thick wave-guiding layers and the relationship between insertion loss and multiple Love wave modes has been elucidated.

\section{ACKNOWLEDGMENTS}

The authors gratefully acknowledge the BBSRC for financial support under research Grant No. 301/E11140. The authors would also like to acknowledge Dr. E. Gizeli and Dr. K. A. Melzak for discussions on the topic of Love waves. 
${ }^{1}$ A. A. Oliner, Acoustic Surface Waves (Springer, Berlin, 1978).

${ }^{2}$ R. M. White and S. W. Wenzel, Appl. Phys. Lett. 52, 1653 (1988).

${ }^{3}$ R. M. White, Faraday Discuss. 107, 1 (1997).

${ }^{4}$ K. K. Kanazawa and J. G. Gordon, Anal. Chim. Acta 175, 99 (1985).

${ }^{5}$ S. Bruckenstein and M. Shay, Electrochim. Acta 30, 1295 (1985).

${ }^{6}$ A. J. Ricco and S. J. Martin, Appl. Phys. Lett. 50, 1474 (1987).

${ }^{7}$ S. J. Martin, A. J. Ricco, T. M. Niemczyk, and G. C. Frye, Sens. Actuators 20, 253 (1989).

${ }^{8}$ F. Josse, Z. A. Shana, D. T. Haworth, S. Liew, and M. Grunz, Sens. Actuators B 9, 97 (1992).

${ }^{9}$ J. Kondoh, S. Shiokawa, and Z. Georgiev, Sens. Actuators B 13-14, 429 (1993).

${ }^{10}$ F. Josse, F. Bender, and R. W. Cernosek, Anal. Chem. 73, 5937 (2001).

${ }^{11}$ M. Rapp, T. Wessa, and H. J. Ache, Ultrason. Symp. Proc. 1-2, 433 (1995).

${ }^{12}$ E. Gizeli, A. C. Stevenson, N. J. Goddard, and C. R. Lowe, IEEE Trans. Ultrason. Ferroelectr. Freq. Control 39, 657 (1992).

${ }^{13}$ G. Kovacs and A. Venema, Appl. Phys. Lett. 61, 639 (1992).
${ }^{14}$ J. Du, G. L. Harding, J. A. Ogilvy, P. R. Dencher, and M. Lake, Sens. Actuators A 56, 211 (1996).

${ }^{15}$ D. P. Morgan, Surface-Wave Devices for Signal Processing (Elsevier, New York, 1991).

${ }^{16}$ G. McHale, M. I. Newton, and F. Martin, Europhys. Lett. (to be published).

${ }^{17}$ G. McHale, M. I. Newton, and F. Martin, J. Appl. Phys. (to be published).

${ }^{18}$ B. A. Auld, Acoustic Fields and Waves in Solids, 2nd ed., (Krieger, Malabar FL, 1990); Vol. II.

${ }^{19}$ M. Rapp (private communication).

${ }^{20}$ M. I. Newton, F. Martin, K. A. Melzak, E. Gizeli, and G. McHale, Electron. Lett. 37, 340 (2001).

${ }^{21}$ M. Weiss, W. Welsch, M. y. Schickfus, and S. Hunklinger, Anal. Chem. 70, 2881 (1998)

${ }^{22}$ D. S. Ballantine, R. M. White, S. J. Martin, A. J. Ricco, E. T. Zellers, G. C. Frye, and H. Wohltjen, Acoustic Wave Sensors (Academic, New York, 1997).

${ }^{23}$ G. McHale, M. I. Newton, F. Martin, E. Gizeli, and K. A. Melzak, Appl. Phys. Lett. 79, 3542 (2001) 